\title{
Crystalline Undulator with a Small Amplitude and a Short Period
}

\author{
Andriy Kostyuk \\ 65933 Frankfurt am Main, Germany
}

\begin{abstract}
The crystalline undulator is a single crystal with periodically bent crystallographic planes. If ultrarelativistic charged particles channel through such a crystal, they emit hard radiation of undulator type. A crystalline undulator with a bending amplitude smaller than the distance between the bent planes and a bending period shorter than the period of channeling oscillations is proposed. Heretofore, it was believed that such a range of bending parameters was unsuitable for a crystalline undulator. This point of view is refuted. In fact, the undulator with a small amplitude and a short period is far superior to what was proposed previously. It requires much lower beam energy for production of photons of the same frequency. Such an undulator allows for a larger effective number of undulator periods. It is predicted to emit intense undulator radiation in the forward direction with a narrow spectral distribution and a lower and softer background. The undulator effect is seen for both positron and electron beams. Using positrons is, however, preferable because they enable one to obtain higher intensity of the undulator radiation with lower background.
\end{abstract}

PACS numbers: $61.85 .+p, 02.70 . \mathrm{Uu}$, 41.75.Fr,41.60.Ap

Synchrotron radiation sources are important tools of modern science. Their applications range from solid state physics, material science, and the semiconductor industry to molecular biology and medicine (see e.g. [1]). The present state-of-the-art sources can emit coherent photons with energy of up to $\sim 10 \mathrm{keV}$. In the regime of spontaneous emission, the photon energy can reach hundreds of $\mathrm{keV}$ [2]. Moving to higher photon energies would be very difficult or even impossible within the present technology. Utilizing the phenomenon of particle channeling [3] may solve this problem: electrostatic fields inside a crystal are very strong and they can undulate trajectories of channeling particles much more effectively than even the best superconductive magnets. Channeling takes place if a particle enters a crystal at a small angle to major crystallographic planes or axes [3]. The particle becomes confined by the (inter)planar or (inter)axial potential and moves along the corresponding plane or axis performing, respectively, transverse oscillations or rotations around the potential minimum. The radiation that is produced due to the transverse motion of a charged projectile is known as the channeling radiation [4]. It has much in common with the undulator radiation, but because the shape of the transverse potential is not parabolic, the motion is not harmonic. As the result, the spectrum of the channeling radiation is broader than that of the undulator radiation, especially in the case of negatively charged projectiles.

It was suggested that a crystal with periodically bent crystallographic planes can be used to obtain radiation of undulator type with a narrower spectral distribution $[5,6]$. In its initial form, the idea of the crystalline undulator assumed that the projectile should follow the sinusoidal shape of the bent crystallographic planes performing channeling oscillations at the same time around the bent central plane of the channel. This implied that the undulator period had to be much larger than the period of channeling oscillations

$$
\lambda_{\mathrm{u}} \gg \lambda_{\mathrm{c}}
$$

Later, a condition of a large amplitude $a_{\mathrm{u}}$ of the crystal bending was proposed [7]

$$
a_{\mathrm{u}} \gg d
$$

Here $d$ is the distance between the bent crystallographic planes that form the channel, i.e. it is the width of the channel. In the following, the crystalline undulator satisfying conditions (11) and (2) is called a large amplitude and long period crystalline undulator (LALP CU). The complete list of conditions that have to be satisfied by the parameters of LALP CU can be found in [8].

It was realized [9] that the dechanneling phenomenon imposes severe restriction on the effective number of periods $N_{\mathrm{u}}$ of the crystalline undulator. The latter should be large, $N_{\mathrm{u}} \gg 1$, to ensure a narrow spectral distribution of the undulator radiation.

The dechanneling takes place due to incoherent collisions of the channeling particle with crystal constituents [3]. Being randomly scattered, the projectile on the average gains transverse energy. If the latter exceeds the height of the interchannel potential barrier, the particle leaves the channel. Starting from this point, it does not follow the shape of the channel and, consequently, it does not emit the undulator radiation. For this reason, the effective number of undulator periods is limited by the average length $L_{d}$ at which the dechanneling ${ }^{1}$ takes

\footnotetext{
${ }^{1}$ Similarly, the effective number of periods is limited by the attenuation of photons in the crystal medium if the attenuation length is smaller than the dechanneling length, $L_{\mathrm{a}}<L_{\mathrm{d}}$. The photon attenuation in $\mathrm{CU}$ was discussed for the first time in [6].
} 
place:

$$
1 \ll N_{\mathrm{u}} \simeq \frac{L_{d}}{\lambda_{\mathrm{u}}},
$$

where $\lambda_{\mathrm{u}}$ is the bending period of the crystalline undulator.

One more condition is relevant to the present discussion. It ensures a stable channeling of the projectile in the bent crystal [6, 10]. It is convenient to write it down in the form ${ }^{2}$

$$
1>C \equiv \frac{F_{\mathrm{cf}}}{U_{\max }^{\prime}}=4 \pi^{2} \frac{a_{\mathrm{u}} E}{\lambda_{\mathrm{u}}^{2} U_{\max }^{\prime}} .
$$

Here $C$ is the centrifugal parameter [8], $F_{\text {cf }}$ is the centrifugal force acting on the projectile in the bent channel, $U_{\max }^{\prime}$ is the maximal force that keeps the particle in the channel and $E$ is the energy of the projectile.

It appears to be difficult to satisfy conditions (2) (44) simultaneously. In particular, they cannot be satisfied in the case of electron beam of moderate energy, $E \lesssim 1 \mathrm{GeV}$. The LALP CU conditions can be fulfilled for positron beams (see [8] and references therein) and for high energy, $E>10 \mathrm{GeV}$, electrons [11]. Nevertheless, the total energy of channeling radiation (integrated over the frequency) exceeds that of the undulator radiation even in these cases (see e.g. figure 8 of [8] or figure 4 of [11]). Moreover, the channeling photons are harder than the undulator ones and, therefore, they cannot be easily screened out. This may cause serious problems for many potential applications.

Let us revisit conditions (2) and (4) and check if they are indeed necessary to construct an intense source of hard photons with a narrow spectral distribution. The further analysis will combine theoretical approach with Monte Carlo simulations using the computer code ChaS (Channeling Simulator). The code performs a 3D simulation of particle trajectories and calculates the spectral and angular distribution of the emitted radiation. In contrast to other channeling codes that either use the continuous potential approximation [12 18 or consider binary collisions of the projectile with the crystal atoms as a whole (ignoring the incoherent collisions with atomic electrons) [19 23], the algorithm of ChaS is based on the binary collisions of the projectile with target electrons as well as with nuclei. This novel feature is especially beneficial in the case of negatively charged projectiles, which channel in the vicinity of the atomic nuclei, where

\footnotetext{
2 The conditions (1), (2) and (4) are tightly related. In particular, (1) can be derived from (2) and (4) 8]. Alternatively, the centrifugal parameter can be expressed in the form $C=R_{\mathrm{c}} / R_{\min }$, where $R_{\mathrm{c}}=E / U_{\max }^{\prime}$ in the critical Tsyganov radius of the channel [10] and $R_{\min }=\lambda_{\mathrm{u}}^{2} /\left(4 \pi^{2} a_{\mathrm{u}}\right)$ is the minimal curvature radius of a sinusoid with the amplitude $a_{\mathrm{u}}$ and the period $\lambda_{\mathrm{u}}$.
}

the continuous potential approximation becomes less accurate and the electron density is much higher than the average one. Results obtained with previous versions of the code were published in [24, 25] and demonstrated reasonable agreement with experimental data. The present version of ChaS uses the electron distribution in the crystal that has been calculated from first principles using the computer code ABINIT [26] and utilizes more efficient algorithms than the previous versions. It takes into account all the physics that is essential for the analysis of the channeling of electrons and positrons with energy $E$ in the range from a few hundreds of $\mathrm{MeV}$ to a few $\mathrm{GeV}$, provided that the photons under interest are not very soft: $\hbar \omega \gtrsim 0.5 \mathrm{MeV}$ and at the same time are not very hard $\hbar \omega \ll E$. The contributions of the phenomena that are not included in the model (e.g. a shift of photon energy due to recoil, losses of the projectile energy due to emission of photons, quantum effects in the motion of the projectile, influence of the crystal medium on the emission, propagation of the radiation) are expected to be small and cannot influence the result substantially [24, 27]. In the following simulations, the emittance of the particle beam was neglected, i.e. the particles were assumed to enter the crystal at zero angle to the crystallographic planes.

Let us reanalyze the reasons behind condition (2). The channel width $d$ sets the upper bound of the amplitude of the channeling oscillations. The amplitude of undulator oscillations has to be much larger than that of the channeling oscillations to make sure that the spectrum is dominated by the undulator radiation rather than by the channeling one. This is, however, necessary only if the frequency of the undulator radiation $\omega_{\mathrm{u}}$ is smaller than the frequency of channeling oscillations $\omega_{\mathrm{c}}$. Indeed, the energy radiated in the forward direction by a moving particle in the case of dipole approximation has the following dependence on the transverse oscillation amplitude $a$ and the radiation frequency $\omega$ :

$$
\left.\frac{d \mathcal{E}}{d \omega d \Omega}\right|_{\theta=0} \sim a^{2} \omega^{4}
$$

where $d \Omega$ is the differential of the solid angle and $\theta$ is the angle between the direction of the radiation emission and the average direction of the particle motion. ${ }^{3}$ One sees from (5) that condition (2) is not necessary; i.e., the amplitude of the undulator bending can be smaller than the channel width,

$$
a_{\mathrm{u}}<d
$$

\footnotetext{
3 The dependence (5) becomes obvious from Larmor's formula for the radiated power applied to the nonrelativistic harmonic oscillations of the projectile in the inertial frame that moves with the average velocity of the particle along the beam direction. Boosting the radiation to the laboratory frame scales the frequency by the factor of $2 \gamma$ but it does not change the proportionality $\sim \omega^{4}$.
} 
provided that the frequency of the undulator radiation is substantially larger than that of the channeling radiation

$$
\omega_{\mathrm{u}} \gg \omega_{\mathrm{c}}
$$

In this case, however, the period of the crystal bending $\lambda_{\mathrm{u}}$ has to be much smaller than the smallest period of channeling oscillations $\lambda_{\mathrm{c}}$ :

$$
\lambda_{\mathrm{u}} \ll \lambda_{\mathrm{c}} \text {. }
$$

The last inequality seems to lead to violation of condition (4). Indeed, the length of the period of the channeling oscillations can be estimated as

$$
\lambda_{\mathrm{c}} \simeq 2 \pi \sqrt{\frac{E}{U^{\prime \prime}(0)}} .
$$

Here $U^{\prime \prime}(0)$ is the second derivative of the transverse potential energy with respect to $y$ in the point of its minimum $y=0$, where the axis $y$ is perpendicular to the channel boundaries. Combining (9) with (8) and taking into account that ${ }^{4}$

$$
U_{\max }^{\prime} \lesssim U^{\prime \prime}(0) d
$$

one obtains from (4)

$$
1>C \gg \frac{a_{\mathrm{u}}}{d} .
$$

The crystal bending amplitude $a_{\mathrm{u}}$ cannot be much smaller than $d$ otherwise it becomes comparable to (or smaller than) the amplitude of thermal vibrations of the crystal atoms. No undulator effect can be observed in this case. If $a_{\mathrm{u}}$ is smaller but comparable to $d$, inequality (11) becomes contradictory. One may think that this should destroy the undulator effect, but, fortunately, it does not happen. Condition (4) is, in fact, irrelevant in the case of a small amplitude (6) and short period (8) (SASP CU).

Simulated trajectories of an electron and a positron channeling in a SASP CU are shown in figure 1, As is seen, the particles do not follow the shape of the bent crystallographic planes. Therefore, formula (4) is not applicable. The channeling process is not destroyed. Roughly, the particle motion can be considered as if it were governed by a continuous potential averaged over the oscillations of the plane. In other words, it is similar to the channeling in a straight crystal with a somewhat different continuous potential.

Nonetheless, a more careful consideration reveals that the particles also perform transverse oscillations with the

\footnotetext{
${ }^{4}$ Expressions (9) and (10) become exact equalities in the case of parabolic potential. For a real potential, the second derivative varies and the maximum value of the force is reached at $|y|<d$, hence (9) is an approximate equality and (10) is an inequality.
}

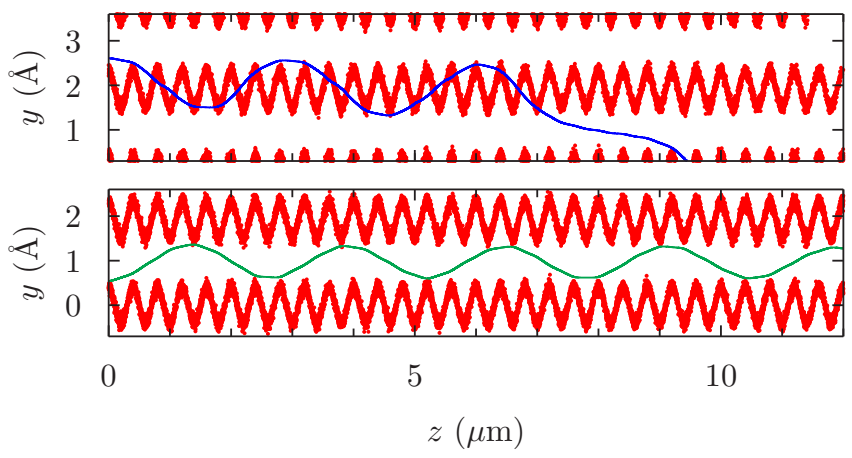

FIG. 1: Simulated trajectories of an electron (upper panel) and a positron (lower panel) with energy $E=855 \mathrm{MeV}$ channeling in a crystalline undulator with a small amplitude, $a_{\mathrm{u}}=0.4 \AA$, and a short period, $\lambda_{\mathrm{u}}=400 \mathrm{~nm}$. The projectile does not follow the shape of the bent crystallographic planes (the thick wavy lines) instead it performs channeling oscillations with roughly the same period as in a straight crystal. The effect of crystal bending on the shape of trajectories is practically unseen.

period $\lambda_{\mathrm{u}}$. The amplitude of these undulator oscillations $a$ is much smaller than the bending amplitude $a_{\mathrm{u}}$. Therefore, it is practically impossible to see the modification of the trajectories due to the crystal bending in figure 1 . Still, the corresponding Fourier harmonics reveal themselves in the spectra of the emitted radiation shown in figures 2 and 3 . Despite that the amplitude $a$ of the undulator oscillations of the projectile is much smaller than that of the channeling oscillations, the undulator radiation peak is enhanced relative to the channeling radiation maximum due to (5) and (7). The undulator peaks are narrow and well separated from the channeling ones. The absolute width of the undulator and channeling radiation peaks is approximately the same. But the relative width of undulator peaks are much smaller due to (7).

It is important to note that there exist technologies that enable one to manufacture a SASP CU. The most mature of them is the growing of $\mathrm{Si}_{1-\chi} \mathrm{Ge}_{\chi}$ crystals [28] with a periodically varying Ge content $\chi$ [29].

The crystal has to be grown in the direction [001] with the atomic fraction $\chi$ of germanium varying between 0 and $2 \bar{\chi}$ according to the formula [30]

$$
\chi(\zeta)=\bar{\chi}\left[1-\sin \left(2 \pi \sqrt{2} \frac{\zeta}{\lambda_{\mathrm{u}}}\right)\right] .
$$

The coordinate $\zeta$ measures the crystal thickness along the the direction of its growth. The beam has to be directed along the bent $(01 \overline{1})$ plane at a small angle to the axis [011]. In this case the beam makes the angle of approximately $45^{\circ}$ with the direction of the crystal growth and, therefore, the bending period of the channel is $\lambda_{\mathrm{u}}$.

The parameter $\bar{\chi}$ in (12) is the average atomic fraction of germanium in the crystal. For desired parameters of 


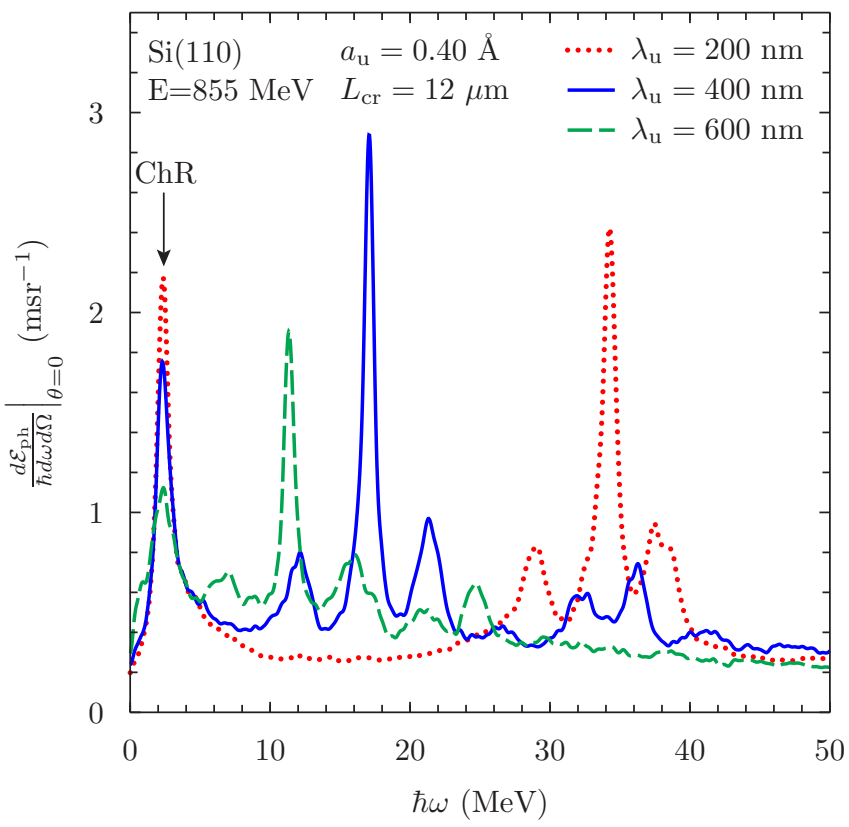

FIG. 2: Simulated spectra of radiation emitted by $E=855$ $\mathrm{MeV}$ electrons channeling in a $12 \mu \mathrm{m}$ long crystalline undulator with a small bending amplitude for three short periods. The spectra are obtained by averaging over about 1000 simulated trajectories. The undulator radiation peaks are higher and are centered at much larger photon energy than the corresponding channeling radiation $(\mathrm{ChR})$ peaks.

TABLE I: The values of the average atomic fraction of germanium $\bar{\chi}$ that correspond to the parameters of the crystalline undulators that have been used in the simulations presented in figures 2 and 3 .

\begin{tabular}{||c||c|c|c||}
\hline \hline \multirow{2}{||c||c||}{$\begin{array}{c}a_{\mathrm{u}} \\
(\AA)\end{array}$} & \multicolumn{3}{|c||}{$\lambda_{\mathrm{u}}(\mathrm{nm})$} \\
\cline { 2 - 4 } & 200 & 400 & 600 \\
\hline \hline 0.4 & 0.034 & 0.017 & 0.011 \\
\hline 0.6 & 0.051 & 0.026 & 0.017 \\
\hline \hline
\end{tabular}

the channel bending $\lambda_{\mathrm{u}}$ and $a_{\mathrm{u}}$, it can be found from the following formula 30 ]

$$
\bar{\chi}=170 \frac{a_{\mathrm{u}}}{\lambda_{\mathrm{u}}}
$$

The values of $\bar{\chi}$ listed in table $\llbracket$ correspond to the undulator parameters that were used in the simulations presented in figures 2 and 3 . It has been shown in [30] that strained layer crystals with such parameters are stable against misfit dislocations.

Hence, the crystalline undulator that violates conditions (2) and (4) can be created. Moreover, it is predicted to be far superior to LALP CU.

Due to small $\lambda_{\mathrm{u}}$, the condition (3) can be satisfied for SASP CU even if it is used with a moderate energy electron beam. This is a big practical advantage since positron sources are much more expensive than electron

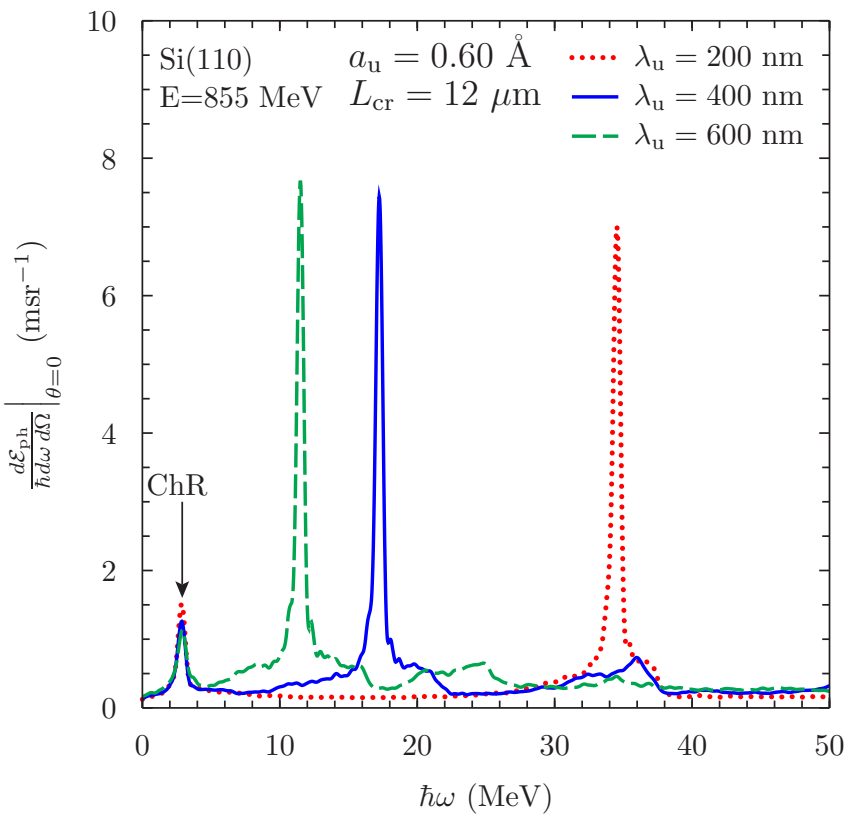

FIG. 3: The same as in figure 2 but for positrons. The undulator radiation peaks are higher than in figure 2 by a factor of $\sim 3$, a better signal-to-background ratio is predicted.

ones, and electron beams are usually of higher quality.

In contrast to the LALP CU, the undulator radiation of SASP CU is much harder than the channeling radiation (7). This a very important feature. A soft photon background is usually easier to get rid of. It is possible to make a detector sensitive to hard photons, but screen it from (or make insensitive to) soft photons. But it is more difficult to do the opposite. This makes SASP CU substantially more suitable for many potential applications in comparison to LALP CU.

Finally, due to much the smaller bending period of SASP CU, it can produce by about 2 orders of magnitude harder photons when used with a beam of the same energy as LALP CU. Or, to put it differently, SASP CU will require a much less expensive accelerator than would be needed for production of photons of the same energy with LALP CU. For the same reason, SASP CU may have an exciting technological potential even in the domain of photon energies around $100 \mathrm{keV}$ where synchrotron radiation sources do exist but are unique and very expensive.

The author is grateful to X. Artru, H. Backe, V. Baryshevsky, S. Dabagov, M. Garattini, V. Guidi, A. Mazzolari, M. Motapothula, A. Shchagin, N. Shul'ga, V. Tikhomirov, and W. Wagner for their interest in the work, for encouraging discussions and critical comments. At its initial stage, the work was supported by Deutsche Forschungsgemeinschaft (DFG). Numerical simulations were done at the Center for Scientific Computing of the J.W. Goethe University, Frankfurt. 
[1] Philip Willmott, An Introduction to Synchrotron Radiation: Techniques and Applications (John Wiley and Sons, New York, 2011).

[2] P. Schmüser, M. Dohlus, J. Rossbach, "Ultraviolet and Soft X-Ray Free-Electron Lasers", Springer, Berlin Heidelberg, (2008).

[3] J. Lindhard, Kong. Danske Vid. Selsk. Mat.-Fys. Medd. 34(14), 1-64 (1965).

[4] M. A. Kumakhov, Phys. Lett. A 57, 17 (1976).

[5] V. V. Kaplin, S. V. Plotnikov and S. A. Vorobiev, Zh. Tekh. Fiz. 50, 1079-1081 (1980), (Sov. Phys. Tech. Phys. 25, 650-651 (1980)).

[6] V. G. Baryshevsky, I. Ya. Dubovskaya and A. O. Grubich, Phys. Lett. 77A, 61-64 (1980).

[7] A. V. Korol, A. V. Solovov and W. Greiner, J. Phys. G 24, L45 (1998).

[8] A. V. Korol, A. V. Solov'yov and W. Greiner, Int. J. Mod. Phys. E 13, 867 (2004).

[9] A. V. Korol, A. V. Solovov and W. Greiner, Int. J. Mod. Phys. E 8, 49 (1999).

[10] E.N. Tsyganov, TM-682, TM-684, Fermilab, Batavia (1976).

[11] M. Tabrizi et al., Phys. Rev. Lett. 98, 164801 (2007).

[12] X. Artru, Nucl. Instrum. Meth. B 48, 278 (1990).

[13] V. Biryukov, Phys. Rev. E 51, 3522 (1995).
[14] V.O. Bogdanov et al., J. Phys.: Conf. Ser. 236, 012029 (2010).

[15] V. Guidi, A. Mazzolari and V. Tikhomirov, J. Appl. Phys. 107, 114908 (2010).

[16] A. V. Korol, A. V. Solovov and W. Greiner, J. Phys. G 27, 95 (2001).

[17] K. Saitoh, J. Phys. Soc. Japan, 54152 (1985).

[18] A.M. Taratin, E.N. Tsyganov and S.A. Vorobiev, Phys. Lett. A 72145 (1979).

[19] M. T. Robinson and O. S. Oen, Appl. Phys. Lett. 2, 30 (1963); Phys. Rev. 132, 2385 (1963).

[20] V.V. Kudrin, Yu. A. Timoshenkov and S.A. Vorobiev, Phys. Stat. Sol. B 58409 (1973).

[21] S. K. Andersen et al., Nucl. Phys. B 167, 1 (1980).

[22] J. F. Bak et al., Nucl. Phys. B 242, 1 (1984).

[23] P.J.M. Smulders and D.O. Boerma, Nucl. Instrum. Meth. B 29, 471 (1987).

[24] A. Kostyuk et al., J. Phys. B. At. Mol. Opt. Phys. 44, 075208 (2011).

[25] A. Kostyuk et al., arXiv:1104.3890 [physics.acc-ph].

[26] X. Gonze et al., Zeit. Kristallogr. 220, 558-562 (2005); Comp. Phys. Commun. 180, 2582-2615 (2009).

[27] U. I. Uggerhøj, Rev. Mod. Phys. 77, 1131 (2005).

[28] M. B. H. Breese, Nucl. Instrum. Meth. B 132, 540 (1997).

[29] U. I. Uggerhøj and E. Uggerhøj, Nucl. Instrum. Meth. B 160, 435 (2000).

[30] A. Kostyuk, arXiv:1301.4491. 